Articles

\title{
Sexual dimorphism of the neuroimmunoendocrine response in the spleen during a helminth infection: a new role for an old player?
}

\author{
Karen Elizabeth Nava-Castro' ${ }^{1}$, Lenin Pavón ${ }^{2}$, Enrique-Becerril'2, María Dolores Ponce- \\ Regalado $^{3}$, Hugo Aguilar-Díaz ${ }^{4}$, Mariana Segovia-Mendoza ${ }^{5}$ and Jorge Morales- \\ Montor $^{6 *}$
}

1 Laboratorio de Genotoxicología y Mutagénesis Ambientales, Departamento de Genotoxicología y Medicina Ambientales, Centro de Ciencias de la Atmósfera, Universidad Nacional Autónoma de México, Ciudad de México, 04510, México

2 Laboratory of Psychoimmunology, National Institute of Psychiatry "Ramón de la Fuente", Calzada México- Xochimilco 101, Colonia San Lorenzo Huipulco, Tlalpan, 14370 Mexico City, Mexico

3 Departamento de Ciencias de la Salud, Centro Universitario de los Altos, Universidad de Guadalajara Av. Rafael Casillas Aceves No. 1200, Tepatitlán, de Morelos, 47610, Jalisco, Mexico.

4 Centro Nacional de Investigaciones Disciplinarias en Salud Animal e Inocuidad, Instituto Nacional de Investigaciones Forestales Agrícolas y Pecuarias (INIFAP), Cuernavaca, Morelos, Mexico

5 Departamento de Farmacología, Facultad de Medicina, Universidad Nacional Autónoma de México, Ciudad de México, México

6 Departamento de Inmunología, Instituto de Investigaciones Biomédicas, Universidad Nacional Autónoma de México, AP 70228, Ciudad de México, 04510, México

* Corresponding authors: Telephone + (5255) 56223854, + (5255) 56223732; Fax number: + (5255)56223369. Emails: jmontor66@biomedicas.unam.mx

\begin{abstract}
The interaction of the nervous, immune, and endocrine systems is crucial in the maintenance of homeostasis in vertebrates, and vital in mammals. The spleen is a key organ that regulates the neuroimmunoendocrine system.-The Taenia crassiceps mouse system is an excellent experimental model to study the complex host-parasite relationship, particularly sex-associated susceptibility to infection. The aim of the present study was to determine the changes in neurotransmitters, cytokines, sex steroids, and sex-steroid receptors in the spleen of cysticercusinfected male and female mice, and the association of these different components with whole parasite counts. We found that parasite load was higher in female in comparison to male mice. The levels of the neurotransmitter epinephrine were significantly decreased in infected male animals. The expression of IL-2 and IL-4 in the spleen was markedly increased in infected mice; however, the expression of Interleukin (IL)-10 and Interferon (IFN)- $\gamma$ decreased. We also observed sex-associated differences between non-infected and infected mice. Interestingly, the data show that estradiol levels increased in infected males but decreased in females. Our studies provide evidence that infection leads to changes on neuroimmunoendocrine molecules in the spleen during infection. These changes are dimorphic and impact the establishment, growth, and reproduction of $T$. crassiceps. Our findings support the key role of the neuroimmune network in determining sexassociated susceptibility to the helminth parasite.
\end{abstract}




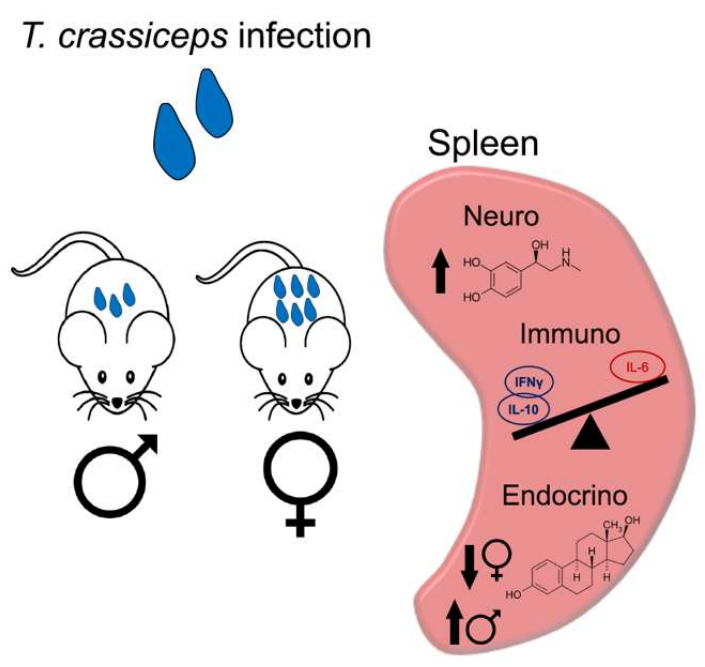

Keywords: Neuroimmunoendocrinology, Spleen, Parasite immunity, Sexual dimorphism, Neurotransmitters, Cytokines, Helminths, Cysticercosis, Taenia crassiceps, Immunity, Infection

\section{Introduction}

Murine intraperitoneal cysticercosis is caused by the helminth cestode Taenia crassiceps.It has been useful to explore the host factors associated with porcine cysticercosis and, to some degree, with human neurocysticercosis. Intraperitoneal T. crassiceps cysticercosis of mice lends itself well to controlled and reproducible experimentation, generating substancial parasite loads in individual mice in a matter of a few weeks after infection [1, 2]. This experimental model allows for a good representation of other forms of cysticercosis because of the parasite's extensive sharing of antigens with other taenias and cestodes. And due to the fact that T. crassiceps and T. solium have high genetic homology $[3,4]$. These characteristics have made murine cysticercosis a convenient instrument to test vaccine candidates and new treatments against cysticercosis, and to evaluate the complex host-cysticercus interaction $[5,6]$. Several features of natural cysticercotic disease have been found by extrapolation from experimental murine cysticercosis [7, 8]. Particularly, experimental murine T. crassiceps cysticercosis has revealed the complexities of the interactive network that regulates infection, formed by the immune and neuroendocrine systems of the host and the parasite [9]. In T. crassiceps cysticercosis, females of all the strains of mice that were studied sustain greater intensities of infection than males. However, during chronic infection (more than 4 weeks), this difference tends to disappear, and the males of the BALB/c strain show a feminization process, characterized by high serum estrogen levels (200 times the normal values) whilst testosterone is $90 \%$ decreased $[10,11]$. At the same time, the cellular immune response (Th1) is markedly diminished in both sexes, and the humoral (Th2) response is enhanced [12]. In addition, estradiol is involved in the immunoendocrine regulation of murine T. crassiceps cysticercosis as a major factor promoting cysticercus growth, through deregulating the thymus-dependent cellular mechanisms that obstruct parasite growth [13]. Furthermore, gonadectomy, which decreases the serum levels to an undetectable level, alters the growth of the parasites, giving similar parasitic loads in both sexes [14] Interestingly, the absence of estrogens does not prevent the growth of the parasites in both genders, demonstrating that although estradiol favors T. crassiceps development, it is not indispensable for rapid parasite growth [11].

Sexual dimorphism (SD) in infections due to parasites is a scarcely studied biological phenomenon of considerable significance for individual health, behavior, and lifestyles, as well as for the evolution of species [9, 15]. Particularly in the hosts, cysticercosis has been more prevalent in females, which carry larger parasite loads, more severe infection and a higher resistance to develop protective immunity to variable degrees. These differences are associated with their genetic background and the duration of the infection [16]. Mechanisms underlying sexual dimorphism in murine cysticercosis consist in a complex network, in which the host's major sex steroids and their receptors, along with other effectors in the immune and central nervous systems, interact with each other and with the parasite [17]. 
For several years, our workgroup has been studying the neuroimmunoendocrine control of T. crassiceps infection [16, $18,19]$. However, neuroimmunoendocrine modulation of T. crassiceps infection in the spleen has never been studied in both sexes. The coordination of the immune system is essential for physiological homeostasis and critical for the proper response to infection [1, 20-23]. At the same time, the unregulated production of molecules from the neuroimmunoendocrine network is one of the principal causes of morbidity and mortality [24-26]. As part of the neuroimmunoendocrine network, the autonomic nervous system connects the immune and endocrine system with the other organs and orchestrates the immune responses according to the physiological needs [27]. Classically, sympathetic and parasympathetic systems act in opposition to maintain physiological homeostasis [28]. Although the sympathetic system has been studied for years, the anti-inflammatory potential of the parasympathetic system was just recently demonstrated [29, 30]. Nevertheless, there is not much evidence about its participation during the infection by $T$. crassiceps.

The aim of this study was to investigate neuroimmunoendocrine changes in the spleen of male and female infected mice with T. crassiceps. and to correlate these data with the parasite burdens. Our results provide evidence that chronic infection leads to persistent changes in neuroimmunoendocrine function in the spleen. These findings support the key role of neuroimmunoendocrine network in sexual dimorphism under T. crassiceps infection.

\section{Results}

2.1 Number of parasites

Figure 1 shows the individual number of parasites recovered from infected mice of both sexes. As expected, we observed a strong sex-associated susceptibility to the infection. Female mice reached a parasite load of $172.3 \pm 44.6$ per mouse at 16 weeks of infection, while male mice showed half the parasite burden than females (985.6 \pm 66.0$)$ (Figure 1). Parasites were found exclusively in the peritoneal cavity of infected mice, and never in any other organ or compartment of the host. Also, mice did not have any apparent sign of disease. The infected mice did not lose weight and the appearance of their fur was maintained throughout the experiment.

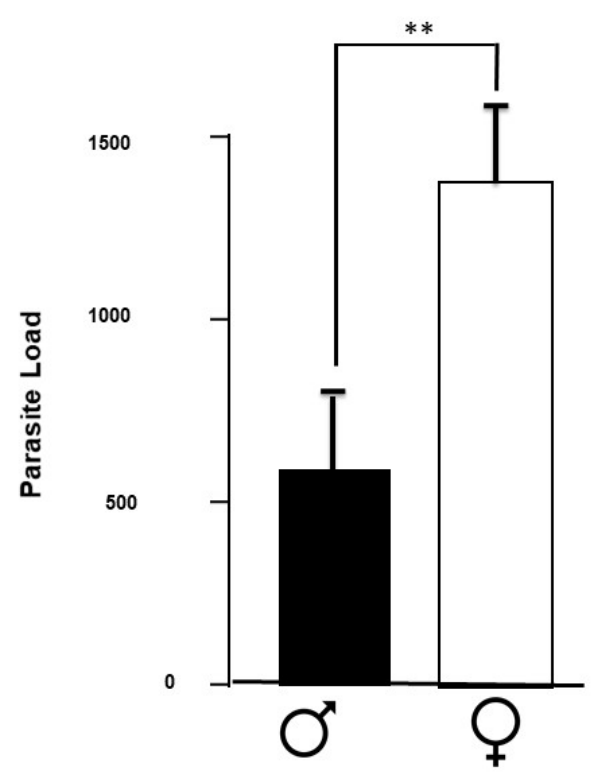

Figure 1. Parasite load in infected mice of both sexes. Data show the number of parasites recovered from the peritoneal cavity of 20 female and 20 male BALB/c mice at 16 weeks post-infection. Each point represents individual parasite loads. ${ }^{* *}, \mathrm{P}<0.001$ when comparing male and female infected mice. 


\subsection{Levels neurotransmitters in the spleen}

Figure 2 shows the splenic concentrations of four neurotransmitters in infected mice of both sexes. As depicted, neurotransmitter levels in infected groups differ from those found in controls except for dopamine (DOPA), which was higher in controls of female mice. The levels of norepinephrine (NE), epinephrine (EP) and serotonin (5-HT) were sexually dimorphic. NE was significantly higher in infected female mice when compared to their male counterparts and controls (Figure 2). In the contrast, the levels of EP were greater in infected male mice, while in the infected females the concentration of this neurotransmitter was minimal. Finally, the levels of 5HT were similar in both male and female infected mice, however, the uninfected males showed significantly superior levels of this neurotransmitter than the uninfected females. In summary, the changes in neurotransmitter content in the spleen of the infected groups were different from those in the non-infected groups.
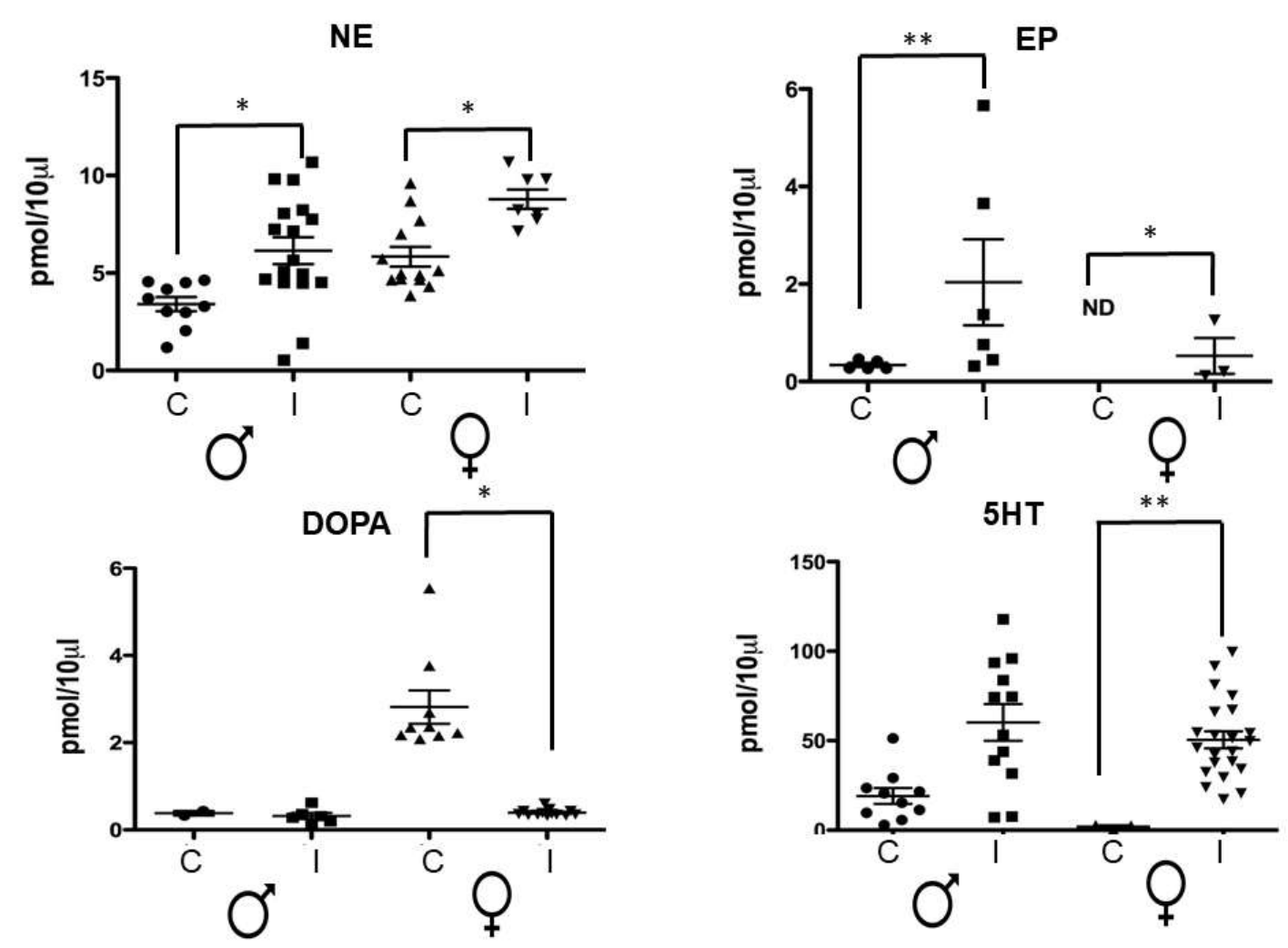

Figure 2. Splenic levels of neurotransmitters in the control groups and in female and male mice infected with T. crassiceps. The levels of NE, EP, DOPA and 5HT were measured in the spleen of the different experimental groups. Data show the levels of neurotransmitters corresponding to 20 female (10 controls and 10 infected) and 20 male (10 controls and 10 infected) BALB/c mice at 16 weeks post-infection $\mathrm{p}<0.05^{*}, \mathrm{p}<0.01^{* *}, \mathrm{p}<0.001^{* * *}$ Abbreviations: NE, norepinephrine; EP, epinephrine; DOPA, dopamine; 5HT, serotonin; C, uninfected control; I, infected.

\subsection{IL-2, IL-4, and IL-10 spleen expression}

The relative expression of IL-2, IL-4 and IL-10 in the spleen obtained from male and female mice in response to $T$. crassiseps infection is shown in (Figure 3). The expression of IL-2 was higher in males in both the control group and infected group as compared with uninfected and infected females. However, the levels of this cytokine were higher in infected males in comparison to its respective control group. IL-4 had an opposite trend to that shown by IL-2. It should be noted that in the group of infected male mice, the levels of IL-4 were significantly higher than in the control 
group; while the levels of this cytokine in infected female mice decreased when compared with their respective uninfected counterpart. Regarding IL-10, the infection diminished its levels in both sexes.
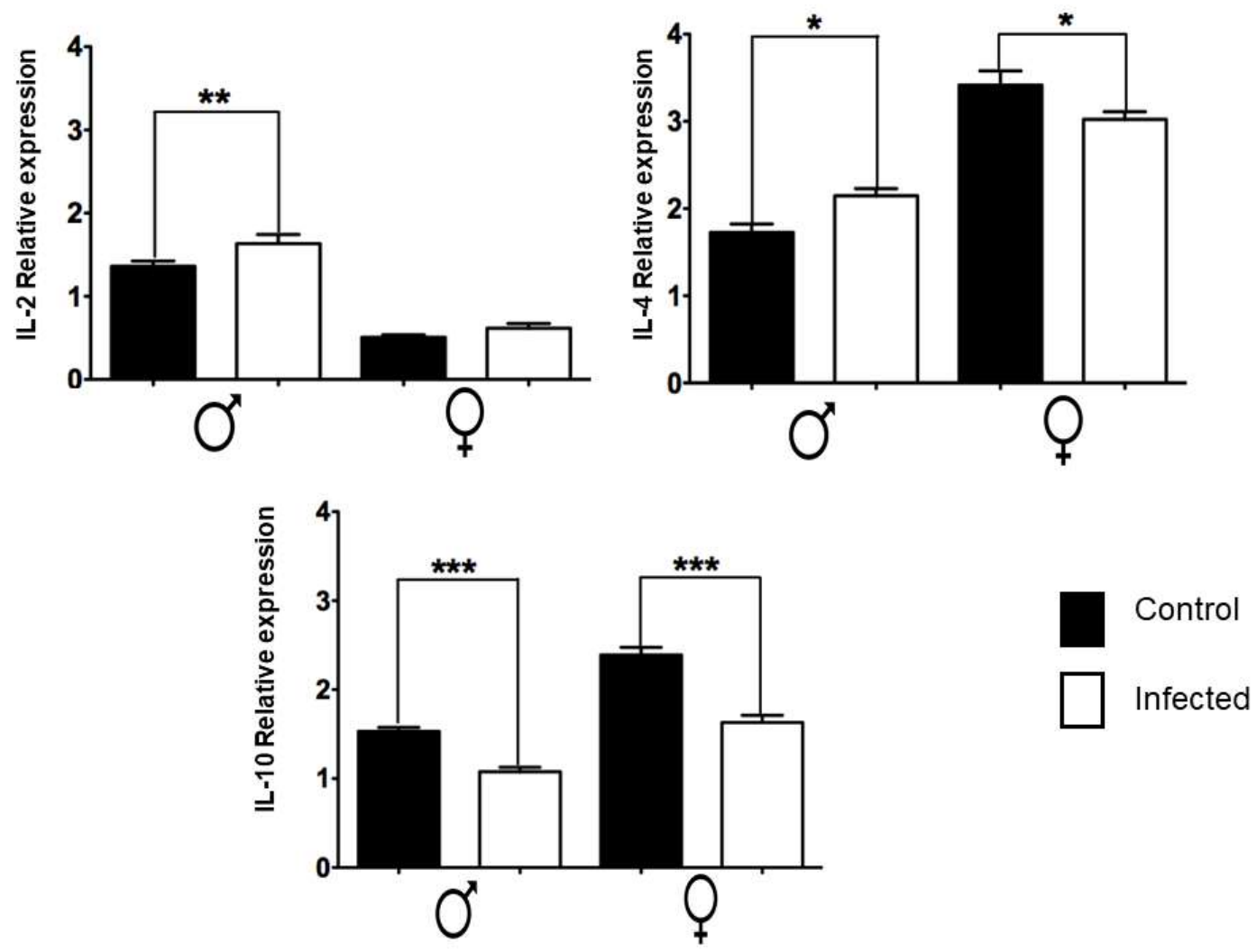

Figure 3. Relative expression of splenic levels of IL-2, IL-4, and IL-10 in the control groups and in male and female mice infected with $T$. crassiceps. Data show the levels of different cytokines corresponding to 20 female (10 controls and 10 infected) and 20 male (10 controls and 10 infected) BALB/c mice at 16 weeks. $\mathrm{p}<0.05^{*}, \mathrm{p}<$ $0.01^{* *}, \mathrm{p}<0.001^{* * *}$

\subsection{IFN- $\gamma$, TNF- $\alpha$ and IL-6 expression in the spleen}

The infection with T. crassiceps induced a significant decrement of IFN- $\gamma$ levels in infected individuals of both sexes as compared to their respective control groups; an opposite effect to that observed with the splenic levels of TNF- $\alpha$ (Figure 4). Regarding the concentration of IL-6, there were no differences between infected and healthy animals, regardless of their sex. Of note, although no significant changes were observed in the levels of this cytokine between sexes, female mice have considerably high levels compared to males. 


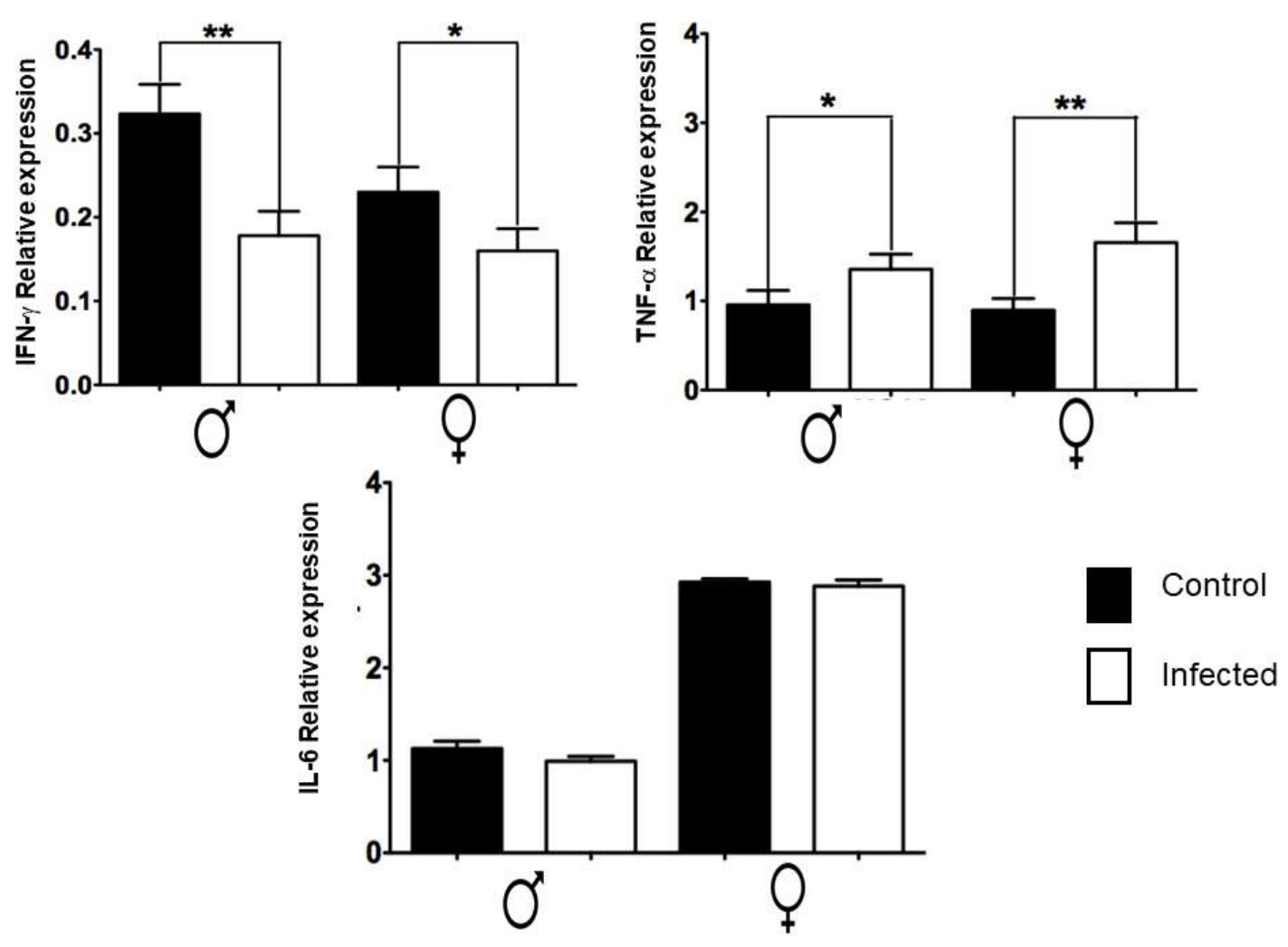

Figure 4. Relative expression of splenic levels of IFN $\gamma$, TNF- $\alpha$, and IL-6 in the control groups and in male and female mice infected with T. crassiceps. Data show the levels of different cytokines corresponding to 20 female (10 controls and 10 infected) and 20 male (10 controls and 10 infected) BALB/c mice at 16 weeks. p $<0.05^{*}, \mathrm{p}<$ $0.01^{* *}, \mathrm{p}<0.001^{* * *}$

\subsection{Sex steroids levels in the spleen}

We measured the splenic content of progesterone, estradiol, and testosterone all groups (Figure 5). Progesterone levels were not modified due to the infection in both sexes (Figure 5). In the case of estradiol, there was a marked increase in its levels in infected male animals, that reached similar levels to those of control females. Conversely, females had a slight reduction of estradiol content in the spleen (Figure 5). The testosterone levels were also altered in infected males and females, while males had a strong reduction in the production of this hormone, females had a slight increase (Figure 5). 


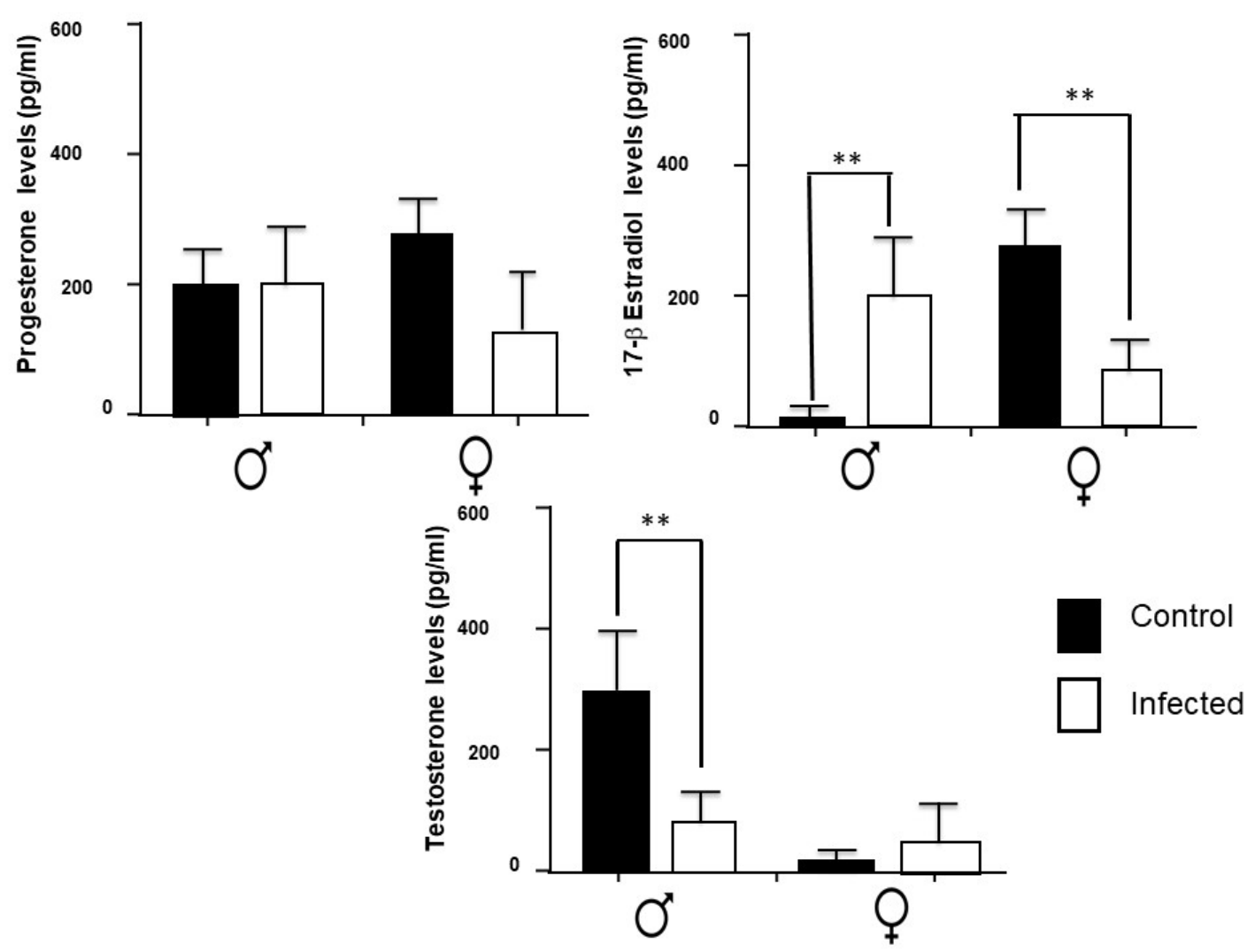

Figure 5. Splenic levels of steroid hormones, progesterone, estradiol, and testosterone, in the control groups and in male and female mice infected with T. crassiceps. Data show the levels of different hormones corresponding to 20 female (10 controls and 10 infected) and 20 male (10 controls and 10 infected) BALB/c mice at 16 weeks post-infection, $\mathrm{p}<0.001,{ }^{* *}$ when comparing the control group and infected individuals. $\mathrm{p}<0.05^{*}$, $\mathrm{p}<0.01^{* *}, \mathrm{p}<0.001^{* * *}$

\subsection{Expression of sex steroid receptors in the spleen}

The expression of nuclear estrogen receptors (ER- $\alpha$ and $\operatorname{Er} \beta$ ), progesterone receptor (PR), and androgen receptor (AR) in the spleen of the mice were measured by western blot (Figure 6). Interestingly, in the infected male mice, there was a slight but significant increment of the expression of both nuclear ERs as compared with the control group, while no changes were observed in the female population. Additionally, the levels of PR were also significant increase in male infected mice; again, no changes were observed in the female counterpart. Of note, the levels of protein expression of ERs and PR in infected male mice exceeded the levels of these molecules found in females. In addition, the expression of AR was significant reduced in both infected male and female mice as compared with each control group. 
A

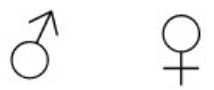

C I C I

ER- $\alpha$

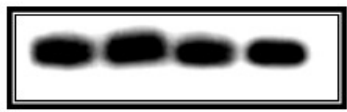

ER- $\beta$

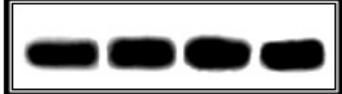

PR

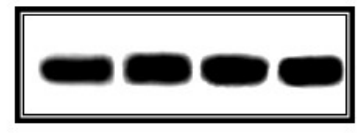

AR

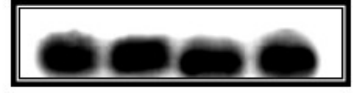

$\beta$-Actin

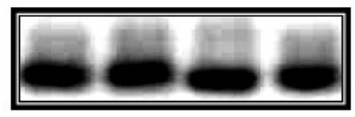

B
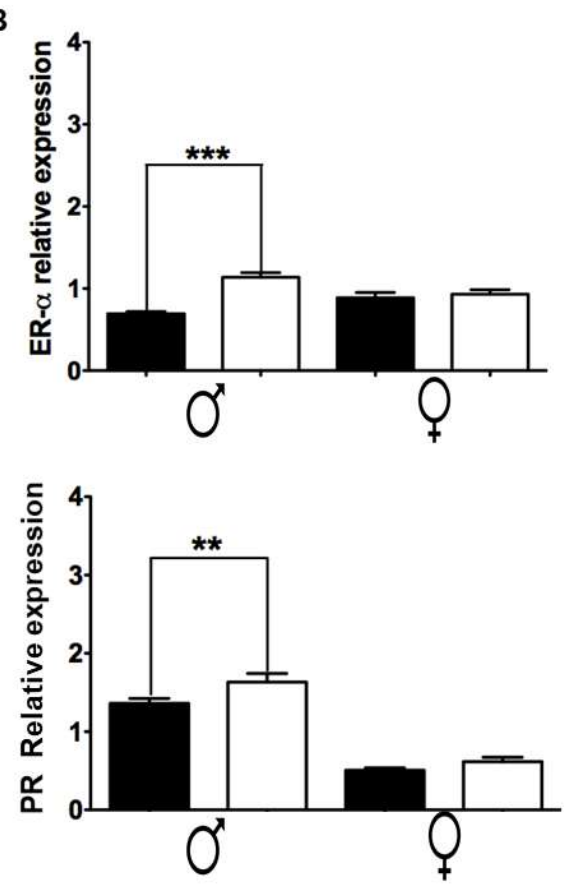
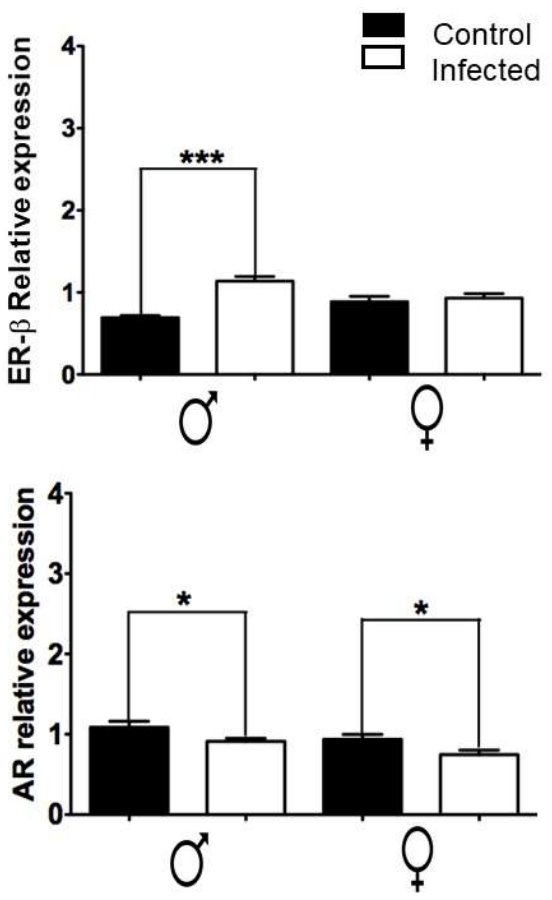

Figure 6. Splenic levels of the different steroid receptors, ER $\alpha, E R \beta, P R$ and $A R$, evaluated in male and female mice non infected and infected with T. crassiceps. A representative image (A) and the densitometry analysis of the different experiments (B) are shown. B-actin was used as the loading control. Normalization of values was done against the loading control. 20 female (10 controls and 10 infected) and 20 male (10 controls and 10 infected) BALB/c mice at 16 weeks were used. 


\section{Discussion}

Few studies have investigated the effects of chronic infection on the neuroimmunoendocrine response in lymphoid organs [28]. The spleen is the largest secondary immune organ and comprises two main compartments: 1) the red pulp, which filters the blood of foreign material and damaged erythrocytes, and 2) the white pulp, which initiates immune reactions to blood-borne antigens [29]. This study provides strong evidence of the interaction between helminth infections and the neuroimmunoendocrine system in the spleen, in mice of both sexes. To our knowledge, this is the first study to demonstrate a relationship between helminth infection and the neuroimmunoendocrinology of the spleen, additionally, we provide evidence that this interaction depends on the sex of the individual.

The interaction of the nervous, endocrine, and immune systems is crucial in the maintenance of homeostasis in vertebrates, and it is vital in mammals [30,31]. The capacity of the immune system to discriminate between self and non-self is based on the high specificity poised by immune cells. The immune response plays a key role in the control of physiology, contributing to maintain the integrity of the body cells and tissues [32]. Hormones and neurotransmitters present in the immune cell microenvironment can restrict their autonomy, probably by acting through their receptors located on immune cells. Efficient communication among these three systems implies the existence of afferent and efferent pathways, constituting a complex feedback system, which, when altered, can trigger different pathologies. [33, 34].

Since the aim of this work was to study these neuroimmunoendocrine interactions, we evaluated biological components of these three systems, i.e., we measured cytokines as indicators of immune function, neurotransmitters as components of the neuronal axis, and hormones, which are involved in the endocrine network. We observed that infected male and female mice had a significant increase of NE and 5HT in the spleen (Fig. 2). In addition, we found a dimorphic response to the infection, since EP was higher in infected males than in infected females.

Serotonin levels have also been reported to increase when an infection is taking place; this increase is associated with the eradication of the disease via multiple pathways [35, 36]. We also observed a significant increase in NE, which other studies have associated to Th2 polarization and M2 macrophage differentiation as well as an increase in nitric oxide (NO) production) [37-39]. In addition, the increase in 5HT is related to T and B lymphocyte proliferation, which enhance the establishment of a humoral response to infection [40, 41].

Cytokines are other soluble factors that are secreted in response to infections [42-44]. Regarding the immune system, we explored the two counterparts involved in inflammation, Thelper type 1 (TH1) and T helper type 2 (TH2). Usually, mammals employ the Thelper type 2 (TH2) immune response to protect against helminth infections [45, 46]. Related to this, we observed that IL-6 was upregulated in healthy and infected female mice. Different reports have demonstrated that the deficiency of this cytokine is related to the absence of parasitic burden, increased immunity, and resistance to the parasite in an H. polygyrus infection mouse model. Moreover, IL- 6 can modulate different lineages of $\mathrm{T}$ lymphocytes, as well as antibody production [45]. In contrast, the upregulation of this cytokine has opposite effects. According to the above, the results presented here agree with this assumption, because elevated levels of IL-6 correlate with a higher more parasitic loads in females as compared to males. Our results are also in accordance with previous reports that mention that IL-4 is increased after intestinal nematode infection [40]. We were only able to corroborate these findings in infected males. Infected females, on the other hand, showed a significant decrease compared to their control group. The foregoing suggests that the infection with $T$. crassiceps is dimorphic, which could be accompanied by the modulation of different immune lineages that need further study.

Interestingly, we observed an increase in estradiol levels in infected males, while females had a decrease in this hormone. These changes were not accompanied by differences in the expression of its nuclear receptors. Additionally, the levels of testosterone in male mice were also negatively affected, in a similar manner to its corresponding receptor. This is in accordance with other studies that report that intracellular protozoan parasite infections can have an impact on sex hormones and the immune response [48, 49]. Therefore, our data also support the existing literature regarding sexual dimorphism in the susceptibility to parasitic diseases. 
The findings presented in this paper must be interpreted considering some limitations. First, our design does not allow for causal inferences. Present and past experimental evidence suggests that host-parasite interactions are associated to changes in neural activity, interleukin and cytokine production, and hormones in the host, which interact with the parasites' survival and reproduction [18, 19, 23, 50. The most important feature of these changes is that they are dimorphic, so the response of each sex determines parasite adaptation and growth.

The possibility that the parasympathetic innervation to the spleen directly senses peritoneal components through its nerve endings cannot be excluded. Considering that: 1) intraperitoneal murine cysticercosis is accompanied by a number of unique events (i.e. a bias towards the female environment, feminization of male hosts, a permissive TH2inclined immune response) $[\mathbf{9}, \mathbf{5 1}$. 2) this infection does not kill the host in spite of the huge parasite loads, and 3) the host's immune response does not totally reject the parasite, one is led to suspect that such host-parasite conviviality rests upon unusually effective mechanisms that enable physiological stability for both host and parasite, whereby the spleen it is a key player.

Finally, our results indicate that parasite stimuli can influence cellular events within the spleen, which is considered one of the most specific peripheral organs of the immune response and suggest that a distinctive feature of the infection is the change in neurotransmitters, cytokines, and hormones, which are involved with the control of parasite growth in a sexually dimorphic fashion. Regardless of how they arise, the changes observed in the spleen of the infected male and female mice may be something more than just an interesting observation: they may also indicate a general host reaction and the involvement of the host-parasite relationship in acquired and innate immune responses, as well as behaviors. Thus, parasite infections can induce systemic alterations such as malnutrition, anemia, and impaired cognitive functions $[\mathbf{5 2}, \mathbf{5 3}]$, which should be considered in a preponderant way.

\section{Materials and Methods}

\subsection{Ethics Statement}

Animal care and experimental practices were conducted at the Unidad de Modelos Biológicos (UMB) at the Instituto de Investigaciones Biomédicas (IIB), Universidad Nacional Autónoma de México. All experimental procedures were approved by the Institutional Care and Animal Use Committee (CICUAL, permit number 2015-00134) adhering to the Mexican regulation (NOM-062-ZOO-1999), and in accordance with recommendations from the National Institute of Health (NIH) of the United States of America (Guide for the Care and Use of Laboratory Animals). The euthanasia of experimental animals was performed by overdose of sevoflurane.

\subsection{Animals and experimental infections}

Male and female Balb/c AnN (H2-d) inbred mice obtained from Harlan (Mexico) were used in all experiments. Animals were reproduced and housed in the animal care facilities at Instituto de Investigaciones Biomédicas, (UNAM), under controlled temperature and a $12 \mathrm{~h}$ dark-light cycle with lights switched on between 0700 and 1900. They were fed Purina Diet 5015 (Purina, St. Louis, MO) and given tap water ad libitum. Puberty onset -vaginal opening- as well as the immediate cyclicity were evaluated in females. The fast-growing ORF strain of T. crassiceps was used for infection in all experiments [54]. Larvae were obtained from infected 3-6-month female donor mice. Ten non-budding T. crassiceps larvae (approximately $2 \mathrm{~mm}$ in diameter) were suspended in $0.3 \mathrm{ml}$ sterile phosphatebuffered saline (PBS: 0.15M NaCl, 0.01M sodium phosphate buffer, $\mathrm{pH} 7.2$ ) and intraperitoneally injected into each of eight to ten 42-day-old male and female mice using a 0.25 -gauge needle. Ten non-infected mice of each sex were used as age-matched controls. Mice were rapidly euthanized by overdose of sevofluorane (Pfizer, Mexico) at 16 weeks of infection. Vaginal smears were previously taken from females to monitor the estrous cycle; when cycling, euthanasia was performed in metaestrous. Peritoneal cysticerci were collected and counted after rinsing the peritoneal cavity with PBS. Spleens were collected immediately after rinsing.

\subsection{Tissues sample}

The spleen of infected and normal mice of both sexes was excised immediately after euthanasia and processed as described. After euthanasia, mice spleens were rapidly removed and chilled in cold $0.9 \%$ saline solution. All spleens were split in 3 parts, that were processed according to the variable to be measured (nervous, immune or endocrine). 


\subsection{Neurotransmitter measurement}

Fresh spleens (a third of the total tissue) were placed in a 1.5-mL microcentrifuge tube on dry ice. Ice-chilled $0.1 \mathrm{M}$ PCA containing internal standard ( $10 \mathrm{mg}$ tissue in $300 \mu \mathrm{L}$ PCA) was added into the tissue tube. Then, the tubes were placed on crushed ice or at $4^{\circ} \mathrm{C}$, until the PCA thawed. Samples were sonicated briefly with a microprobe fitted in the sample tube (6-7 s, duty cycle $80 \%$, output control 3) until the tissue was completely homogenized. The tubes were kept on crushed ice or at $4^{\circ} \mathrm{C}$ for $10 \mathrm{~min}$. After a short vortex of samples, $30 \mu \mathrm{L}$ of homogenate were set apart for the protein assay. Samples were then centrifuged at $14,000 \mathrm{rpm}(18,000 \times \mathrm{g})$ for $15 \mathrm{~min}$, at $4^{\circ} \mathrm{C}$. The supernatant was transferred into another clean 1.5-mL microcentrifuge tube, which was. The supernatant was used to determine neurotransmitters (serotonin, dopamine, and other monoamines) for HPLC assay. The system, in sequence, consists of a pump, a pulse damper, an autosampler, a PEEK in-line filter, a column, a conditioning cell, and analytical cells. Prior to HPLC analysis, the fresh mobile phase was passed through the system overnight in a recycling mode. The system parameters were as follows: flow rate: $1.0 \mathrm{~mL} / \mathrm{min}$, column oven temperature: $30^{\circ} \mathrm{C}$, injection volume: $15 \mu \mathrm{L}$, autosampler sample chamber temperature: $4^{\circ} \mathrm{C}$, applied potentials $(\mathrm{mV})$ : conditioning cell $=+10$; analytical cell, E1 $=+50$; $\mathrm{E} 2=+340$. Once the system was set up and equilibrated, the concentration series of the standard mixture of monoamines and their metabolites were assessed, to make sure a clear chromatographic separation was obtained. Also, the individual standards to identify each peak in the mixture by its retention time and voltametric response were carried out. Readings of peak height and peak area were obtained by using the analytical system software. Sensitivity was determined by cutting off at the ratio of signal peak when the noise peak was lower than 5 for every monoamine or metabolite. An internal standard was used for plotting the standard curve of each monoamine and metabolite; and their least-squares linear regression equations were obtained by using Microsoft Office Excel. Concentrations of monoamines and their metabolites in the sample were calculated by using standard equations. All the monoamines and their metabolites in samples were well eluted in <18 min; and afterward, the baseline did not show more interfering peaks. The order and retention time of the eluted monoamines and their metabolites as seen in the chromatogram were: NE (2.38 min), DOPAC (4.17 min), DA (5.29 min), 2,3-DHBA (6.99 min), 5-HIAA (7.61 $\mathrm{min})$, HVA (9.83 $\mathrm{min})$, and 5-HT (14.9 $\mathrm{min})$.

\subsection{Cytokine spleen expression}

Spleen cytokines were measured with the ABTS ELISA kit (Cat\# 900-K00). Antibodies for IL-6, IL-1 IL-10, TNF- $\alpha$, IFN- $\gamma$ and unconjugated antibodies were used for cytokine capture according to the manufacturer's instructions, with a few modifications. Briefly, coated plates (96-well plate, MaxiSorp Nunc Cat. NNC\#442404) were incubated with $50 \mu \mathrm{l}(2 \mu \mathrm{g} / \mathrm{ml})$ of different antibodies overnight. After rinsing 3 times, (Wash buffer, PeproTech), the plates were blocked (Block buffer, PeproTech) and then washed again. Fifty $\mu$ l of sera (1:2 dilution) or tissue protein (10 $\mu \mathrm{g}$ ) were added to the plate in duplicates (in diluent solution, PeproTech). The samples were maintained at $4^{\circ} \mathrm{C}$ for 2 hand washed three times. An enzyme-substrate reaction was developed with ABTS liquid substrate (PeproTech). All solutions were obtained from the ABTS ELISA buffer kit. The plates were read at a wavelength of $405 \mathrm{~nm}$ with a wavelength correction set at $650 \mathrm{~nm}$ at different time points in a Stat Fax 4200 microplate reader (Awareness Technology).

\subsection{Sex steroids levels in the spleen}

Freshly extracted spleens were homogenized in PBS. Steroids were ether-extracted as previously reported [11] and solubilized in the phosphate buffer used for Enzyme-immunoassay (EIA). Estradiol, testosterone and dihydrotestosterone levels were detected using EIA DetectX® kits (Arbor Assays, Ann Arbor MI), according to manufacturer's protocol.

\subsection{Expression of steroids receptors (ER, PR, and AR) in the spleen by western blot}

In brief, as mentioned before, spleen proteins were extracted and disrupted in Tris- $\mathrm{HCl}(1 \mathrm{ml} / 0.1 \mathrm{~g}$ tissue $)$ with a protease-inhibitor cocktail (Calbiochem). The supernatant was recovered after 30 min of centrifugation at 12,000g. The pellet was discarded. Protein concentration was obtained by absorbance at $580 \mathrm{~nm}$ using the Bradford-Lowry method. Total protein of every analyzed tissue $(80 \mathrm{lg}$ ) was boiled in reducing Laemmli sample buffer, separated by SDS-PAGE (8\% acrylamide) and electroblotted onto nitrocellulose membranes. Membranes were blocked for $1 \mathrm{~h}$ in SuperBlock T20 (Thermo Scientific, USA). For protein immunodetection in splenocytes, membranes were subjected 
to overnight immunoblotting with $1 \mathrm{lg} / \mathrm{ml}$ of polyclonal anti-ER $\alpha$ antibodies (HC-20, Santa Cruz Biotech SC-543); ERb (Y-19, Santa Cruz Biotech SC-6821); PR-A/PR-B (C-20, Santa Cruz Biotech SC-539); a-Tubulin (HC20a-543, Sigma T-9026). All antibodies were diluted 1:1000. After incubation with the primary antibody, samples were incubated with HRP-conjugated anti-mouse, anti-goat or anti-rabbit IgG antibodies (Santa Cruz Biotechnology; diluted 1:10000) for $1 \mathrm{~h}$, at room temperature. Next, membranes were washed three times in TBST buffer (10 mM Tris-HCl, $\mathrm{pH} 7.4,100 \mathrm{mM} \mathrm{NaCl}, 0.5 \%$ Tween 20) and the bands were visualized using the ECL system according to the manufacturer's instructions (Super Signal ECL, Pierce). Chemiluminescent signals were captured in Kodak Bio-Max Gel-Doc system film, and the bands were quantitatively analyzed from the digitized image using the Bio-Rad Quantity One software (Bio-Rad, Richmond). Mouse uterus was used as positive control of steroid receptor expression in this experiment. A single band was detected for ER $\alpha$ at $66 \mathrm{kDa}$, ER $\beta$ at $55 \mathrm{kDa}$ and $\beta$-Tubulin at 55 $\mathrm{kDa}$, and two bands for PR at $110 \mathrm{kDa}$ for PR-B and $85 \mathrm{kDa}$ for PR-A. Sex steroid receptor expression level was normalized respective to $\beta$-Tubulin.

\subsection{Experimental design and statistical analysis}

We designed a two-factorial experiment. The independent variable was: 1) infection (two levels: Yes, No). 2) Gender (two levels: male or female). The dependent variables were the number of parasites, the levels of each cytokine (IL2, IL-4, IL-6, L-10, IFN- $\gamma$ and TNF- $\alpha$ in spleen, the levels of neurotransmitters, the levels of sex steroids and expression of sex steroid receptors. The complete design was repeated twice, and the tissues used in each experiment at each time of infection were those from five normal or five infected mice $(\mathrm{N}=10)$. Statistical analysis of Variance Components was performed with the software Prism 2.01 (GraphPad Software). When applicable, post hoc individual contrasts of group mean by the Tukey test used the sum of Residual and Three Factor Interactions variance were performed to test for significant differences.

Author Contributions: Conceptualization JMM and KENC; methodology, KENC, LP, EBV, MDPR, HAD.; software, JMM and KENC.; validation, MSM, MDPR, HAD and EBV; formal analysis, JMM, LP, MSM, HAD, EBV, MDPR.; investigation, KENC, LP, EBV, MDPR, HAD.; resources, JMM.; data curation, JMM.; writing-original draft preparation, KENC, MSM and JMM.; writing-review and editing, MSM and JMM.; supervision, JMM.; project administration, JMM.; funding acquisition, LP and JMM. All authors have read and agreed to the published version of the manuscript.

Conflicts of Interest: The authors declare no conflict of interest.

\section{Acknowledgments / Funding}

This work was partially supported by research grants from the Programa de Apoyos a Proyectos de Investigación e Innovación Tecnológica (PAPIIT) from Dirección General de Asuntos del Personal Académico (DGAPA), UNAM (Grant IN-209719) and grant from CONACYT, FC-2016-2125, both to JMM. 


\section{References}

1. Larralde, C., et al., Experimental cysticercosis by Taenia crassiceps in mice: factors involved in susceptibility. Acta Leiden, 1989. 57(2): p. 131-4.

2. Larralde, C., et al., Immunodiagnosis of human cysticercosis in cerebrospinal fluid. Antigens from murine Taenia crassiceps cysticerci effectively substitute those from porcine Taenia solium. Arch Pathol Lab Med, 1990. 114(9): p. 926-8.

3. Rishi, A.K. and D.P. McManus, Molecular cloning of Taenia solium genomic DNA and characterization of taeniid cestodes by DNA analysis. Parasitology, 1988. 97 ( Pt 1): p. 161-76.

4. Larralde, C., et al., Reliable serology of Taenia solium cysticercosis with antigens from cyst vesicular fluid: ELISA and hemagglutination tests. Am J Trop Med Hyg, 1986. 35(5): p. 965-73.

5. Sciutto, E., et al., New approaches to improve a peptide vaccine against porcine Taenia solium cysticercosis. Arch Med Res, 2002. 33(4): p. 371-8.

6. Sciutto, E., et al., Cysticercosis vaccine: cross protecting immunity with T. solium antigens against experimental murine T. crassiceps cysticercosis. Parasite Immunol, 1990. 12(6): p. 687-96.

7. Morales, J., et al., Castration and pregnancy of rural pigs significantly increase the prevalence of naturally acquired Taenia solium cysticercosis. Vet Parasitol, 2002. 108(1): p. 41-8.

8. Sciutto, E., et al., Improvement of the synthetic tri-peptide vaccine (S3Pvac) against porcine Taenia solium cysticercosis in search of a more effective, inexpensive and manageable vaccine. Vaccine, 2007. 25(8): p. 1368-78.

9. Morales-Montor, J., et al., Host gender in parasitic infections of mammals: an evaluation of the female host supremacy paradigm. J Parasitol, 2004. 90(3): p. 531-46.

10. Meneses, G., et al., Taenia crassiceps cysticercosis: variations in its parasite growth permissiveness that encounter with local immune features in BALB/c substrains. Exp Parasitol, 2009. 123(4): p. 362-8.

11. Larralde, C., et al., Sex hormone changes induced by the parasite lead to feminization of the male host in murine Taenia crassiceps cysticercosis. J Steroid Biochem Mol Biol, 1995. 52(6): p. 575-80.

12. Terrazas, L.I., et al., Shift from an early protective Th1-type immune response to a late permissive Th2-type response in murine cysticercosis (Taenia crassiceps). J Parasitol, 1998. 84(1): p. 74-81.

13. Terrazas, L.I., et al., A role for 17-beta-estradiol in immunoendocrine regulation of murine cysticercosis (Taenia crassiceps). J Parasitol, 1994. 80(4): p. 563-8.

14. Huerta, L., et al., Immunological mediation of gonadal effects on experimental murine cysticercosis caused by Taenia crassiceps metacestodes. J Parasitol, 1992. 78(3): p. 471-6.

15. McLennan, D.A. and D.R. Brooks, Parasites and sexual selection: a macroevolutionary perspective. Q Rev Biol, 1991. 66(3): p. 255-86.

16. Fragoso, G., et al., Preferential growth of Taenia crassiceps cysticerci in female mice holds across several laboratory mice strains and parasite lines. J Parasitol, 2008. 94(2): p. 551-3.

17. Hernandez-Bello, R., et al., Beyond the reproductive effect of sex steroids: their role during immunity to helminth parasite infections. Mini Rev Med Chem, 2012. 12(11): p. 1071-80.

18. Escobedo, G., et al., Molecular mechanisms involved in the differential effects of sex steroids on the reproduction and infectivity of Taenia crassiceps. J Parasitol, 2004. 90(6): p. 1235-44. 
19. Escobedo, G., et al., Parasite regulation by host hormones: an old mechanism of host exploitation? Trends Parasitol, 2005. 21(12): p. 588-93.

20. Rankin, L.C. and D. Artis, Beyond Host Defense: Emerging Functions of the Immune System in Regulating Complex Tissue Physiology. Cell, 2018. 173(3): p. 554-567.

21. MacDonald, A.S., M.I. Araujo, and E.J. Pearce, Immunology of parasitic helminth infections. Infect Immun, 2002. 70(2): $p$. 427-33.

22. Davidson, R.A., Immunology of parasitic infections. Med Clin North Am, 1985. 69(4): p. 751-8.

23. Morales-Montor, J. and C. Larralde, The role of sex steroids in the complex physiology of the host-parasite relationship: the case of the larval cestode of Taenia crassiceps. Parasitology, 2005. 131(Pt 3): p. 287-94.

24. Dantzer, R., Neuroimmune Interactions: From the Brain to the Immune System and Vice Versa. Physiol Rev, 2018. 98(1): $p$. 477-504.

25. McCorry, L.K., Physiology of the autonomic nervous system. American journal of pharmaceutical education, 2007. 71(4): $p$. 78-78.

26. Ulloa, L., The cholinergic anti-inflammatory pathway meets microRNA. Cell Research, 2013. 23(11): p. 1249-1250.

27. Ulloa, L., The Anti-Inflammatory Potential of Selective Cholinergic Agonists. Shock, 2011. 36(1).

28. Arteaga, M., A. Chavarria, and J. Morales Montor, [Immunoneuroendocrine communication network and homeostasis regulation: the use of hormones and neurohormones as immunotherapy]. Rev Invest Clin, 2002. 54(6): p. 542-9.

29. Lewis, S.M., A. Williams, and S.C. Eisenbarth, Structure and function of the immune system in the spleen. Science immunology, 2019. 4(33): p. eaau6085.

30. Bottasso, O. and J. Morales-Montor, Neuroimmunomodulation during infectious diseases: mechanisms, causes and consequences for the host. Neuroimmunomodulation, 2009. 16(2): p. 65-7.

31. Perez, A.R., O. Bottasso, and W. Savino, The impact of infectious diseases upon neuroendocrine circuits. Neuroimmunomodulation, 2009. 16(2): p. 96-105.

32. Jiang, H. and L. Chess, How the immune system achieves self-nonself discrimination during adaptive immunity. Adv Immunol, 2009. 102: p. 95-133.

33. Segovia-Mendoza, M. and J. Morales-Montor, Immune Tumor Microenvironment in Breast Cancer and the Participation of Estrogen and Its Receptors in Cancer Physiopathology. Front Immunol, 2019. 10: p. 348.

34. Kerage, D., et al., Interaction of neurotransmitters and neurochemicals with lymphocytes. Journal of Neuroimmunology, 2019. 332: p. 99-111.

35. Arreola, R., et al., Immunomodulatory Effects Mediated by Serotonin. J Immunol Res, 2015. 2015: p. 354957.

36. Kumar, A., et al., The Serotonin Neurotransmitter Modulates Virulence of Enteric Pathogens. Cell Host Microbe, 2020. 28(1): p. 41-53 e8.

37. Alonso-Trujillo, J., et al., Nitric oxide contributes to host resistance against experimental Taenia crassiceps cysticercosis. Parasitol Res, 2007. 100(6): p. 1341-50.

38. Fuchs, B.A., K.S. Campbell, and A.E. Munson, Norepinephrine and serotonin content of the murine spleen: its relationship to lymphocyte beta-adrenergic receptor density and the humoral immune response in vivo and in vitro. Cell Immunol, 1988. 117(2): p. 339-51. 
39. Grailer, J., M. Haggadone, and P. Ward, Catecholamines promote an M2 macrophage activation phenotype (P1299). The Journal of Immunology, 2013. 190(1 Supplement): p. 63.7.

40. Murray, K., et al., Neuroanatomy of the spleen: Mapping the relationship between sympathetic neurons and lymphocytes. PLoS One, 2017. 12(7): p. e0182416.

41. Herr, N., C. Bode, and D. Duerschmied, The Effects of Serotonin in Immune Cells. Frontiers in cardiovascular medicine, 2017. $4:$ p. 48-48.

42. Ishikawa, N., et al., Early cytokine responses during intestinal parasitic infections. Immunology, 1998. 93(2): p. $257-263$.

43. Hewitson, J.P., J.R. Grainger, and R.M. Maizels, Helminth immunoregulation: the role of parasite secreted proteins in modulating host immunity. Molecular and biochemical parasitology, 2009. 167(1): p. 1-11.

44. Martínez-Saucedo, D., et al., Taenia crassiceps-Excreted/Secreted Products Induce a Defined MicroRNA Profile that Modulates Inflammatory Properties of Macrophages. J Immunol Res, 2019. 2019: p. 2946713-2946713.

45. Patel, N., et al., Characterisation of effector mechanisms at the host:parasite interface during the immune response to tissuedwelling intestinal nematode parasites. Int J Parasitol, 2009. 39(1): p. 13-21.

46. Henry, E.K., J.M. Inclan-Rico, and M.C. Siracusa, Type 2 cytokine responses: regulating immunity to helminth parasites and allergic inflammation. Current pharmacology reports, 2017. 3(6): p. 346-359.

47. Dienz, O. and M. Rincon, The effects of IL-6 on CD4 T cell responses. Clinical immunology (Orlando, Fla.), 2009. 130(1): p. 27-33.

48. Roberts, C.W., W. Walker, and J. Alexander, Sex-associated hormones and immunity to protozoan parasites. Clinical microbiology reviews, 2001. 14(3): p. 476-488.

49. Klein, S.L., Hormonal and immunological mechanisms mediating sex differences in parasite infection. Parasite Immunol, 2004. 26(6-7): p. 247-64.

50. Morales-Montor, J., et al., Taenia crassiceps: androgen reconstitution of the host leads to protection during cysticercosis. Exp Parasitol, 2002. 100(4): p. 209-16.

51. Morales-Montor, J., et al., Immunoendocrine interactions during chronic cysticercosis determine male mouse feminization: role of IL-6. J Immunol, 2001. 167(8): p. 4527-33.

52. Bechir, M., et al., Parasitic Infections, Anemia and Malnutrition Among Rural Settled and Mobile Pastoralist Mothers and Their Children in Chad. EcoHealth, 2012. 9(2): p. 122-131.

53. Carpio, A., et al., Parasitic diseases of the central nervous system: lessons for clinicians and policy makers. Expert review of neurotherapeutics, 2016. 16(4): p. 401-414.

54. Dorais, F.J. and G.W. Esch, Growth rate of two Taenia crassiceps strains. Exp Parasitol, 1969. 25(1): p. 395-8. 